\title{
Elastohydrodynamic Lubrication Analysis of Gear Tooth Surfaces From Micropitting Tests
}

\section{R. W. Snidle}

Cardiff School of Engineering, Cardiff CF24 3TA, UK

\section{N. A. Hopkinson \\ M. Talks}

\section{J. M. Starbuck \\ QinetiQ Ltd, Farnborough, GU14 0LX, UK}

The paper presents numerical results for the elastohydrodynamic lubrication of gear teeth using real surface roughness data taken from micropitting tests carried out on an FZG gear testing machine. Profiles and load conditions corresponding to four load stages in the micropitting test protocol are considered. Elastohydrodynamic film thickness and pressure analyses are presented for conditions having a slide/roll ratio of 0.3 during the single tooth contact phase of the meshing cycle. Comparisons are also included showing the elastohydrodynamic response of the tooth contacts at different times in the meshing cycle for one of the load stages. The rheological model adopted is based on Ree-Eyring nonNewtonian shear thinning, and comparisons are also included of models having constant and different pressure-dependent specifications of the Eyring shear stress parameter $\tau_{0}$. Parameters obtained from the micro EHL analyses are presented that quantify the degree of adversity experienced by the surfaces in elastohydrodynamic contact. These quantify extreme pressure behavior, extreme proximity of surfaces, and pressure cycling within the overall contact and indicate that the different fluid models considered lead to significantly different pressure and film thickness behavior within the contact.

[DOI: $10.1115 / 1.1510881]$

\section{Introduction}

Under ideal conditions of load, speed and oil viscosity, and with very smooth surfaces, gears may operate with a full elastohydrodynamic (EHL) film, the thickness of which may be calculated from the classical formula of Dowson and Higginson. In practice, however, the surfaces of even good quality gears finished by grinding have roughness features that are often far greater in height than the predicted EHL film thickness. Many gears consequently operate under conditions described as "mixed" lubrication or micro EHL. These terms imply significant interaction of the roughness asperities of the two surfaces, or even penetration of the film resulting in a degree of solid contact. A conventional measure of the severity of lubrication is provided by the lambdaratio, which is defined as the mean oil film thickness divided by the combined RMS roughness of the two surfaces. Because of their roughness many practical gears operate with a lambda-ratio significantly less than unity and, under these conditions, theoretical EHL analysis of real gear contacts, which could help provide a better understanding of tooth surface distress phenomena such as scuffing and micropitting, has been found to present a considerable challenge. The presence of roughness on both of the tooth surfaces, which move relative to their instantaneous contact, necessitates a time-dependent treatment. In addition the shear rates imposed on the lubricant separating the sliding asperity micro contacts are sufficiently high to take the lubricant well into the non-Newtonian regime of EHL that has been reported from the experimental work of numerous authors $[1,2,3]$.

EHL analysis of rough surfaces in general has been the objective of numerical modellers over the last decade in attempting to build an understanding of the surface failure modes occurring in real contacts, but numerical models have tended to be limited to lambda-values in excess of unity, where little surface roughness interaction takes place. Zhu and co-workers [4,5] were among the first to develop a "mixed" lubrication EHL model for elliptical

Contributed by the Tribology Division of THE AMERICAN SOCIETY OF MECHANICAL ENGINEERS for presentation at the ASME/STLE Tribology Conference, Cancun, Mexico October 27-30, 2002. Manuscript received by the Tribology Division February 19, 2002 revised manuscript received July 20, 2002. Associate Editor: A. A. Lubrecht. contacts in which films below an arbitrary low value are regarded as creating contacts with a corresponding simplifying modification of the Reynolds equation presumed at the contacting areas. Such analyses have predicted a considerable degree of direct surface contact [6], which is said by the authors to be insensitive to the (small) film level at which contact is taken to occur. The treatment of contact in this model is somewhat arbitrary however, and the assumptions made in dealing with contact remain to be verified.

In contrast the current contribution to rough surface EHL research is based on a theoretical model for line contacts (the appropriate configuration for spur gears) that solves the elastic and hydrodynamic film thickness equations simultaneously using a consistent, mass-conserving, fully coupled method. This has enabled results to be obtained for extremely low lambda values with contact, if it occurs, established directly from solution of the timedependent equations. A second important feature that emerges using this approach, dependent upon the oil rheology model, is that of lubricant cavitation under severe conditions of thin films/high roughness [7].

The paper presents the results of applying the above analysis technique to a set of profiles corresponding to the later (most heavily loaded) stages in an FZG gear test. The results form part of an investigation program that compares the micropitting performance of test gears using two different oils in both simple spur configuration in the FZG tests considered in this paper, and in more sophisticated helical gear testing. Use of experimental gear tooth profiles under the real operating conditions of the gears is judged to be an important requirement as surface profile topography is significantly modified by running-in, and engineering interest must be primarily focussed on the stress and film thickness response actually encountered by the components.

\section{Theory}

The EHL model is expressed in terms of two fundamental relationships, the Reynolds equation for the lubricant relating its pressure with the fluid film thickness, and the elastic deflection of the surfaces under the action of pressurised lubricant. The Reynolds equation is expressed as 


$$
\frac{\partial(\rho h)}{\partial t}+\frac{\left(U_{1}+U_{2}\right)}{2} \frac{\partial(\rho h)}{\partial x}-\frac{\partial}{\partial x}\left(\frac{\rho h^{3}}{12 \eta} S \frac{\partial p}{\partial x}\right)=0
$$

and, in discretised form, the elastic equation is obtained as

$$
\frac{\partial^{2} h\left(x_{j}\right)}{\partial x^{2}}=\sum_{\text {allk }} f_{k-j} p_{k}+\frac{1}{R}+\frac{\partial^{2} \phi}{\partial x^{2}}
$$

This differential form adopted for the elastic deflection enables Eqs. (1) and (2) to be solved simultaneously [7]. This task is made possible by the rapid decay of the influence coefficients $f_{i}$ as the index $i$ increases from zero [8] in comparison with the influence coefficients obtained in discretising the conventional integral deformation equation.

The non-Newtonian factor $S$ depends on pressure, film thickness, sliding speed and pressure gradient. For the Eyring shearthinning model it is available in closed form [9] as Eq. (3) and this model is used in the current treatment

$$
\begin{gathered}
S=\frac{3(\Sigma \cosh \Sigma-\sinh \Sigma)}{\Sigma^{3}} \sqrt{1+\frac{\eta^{2}\left(U_{2}-U_{1}\right)^{2}}{\tau_{0}^{2} h^{2}} \frac{\Sigma^{2}}{\sinh ^{2} \Sigma}} \\
\text { where } \Sigma=\frac{h}{2 \tau_{o}} \frac{d p}{d x}
\end{gathered}
$$

The dependence on pressure of oil viscosity and density are taken to be given by the well known Roelands (4) and Dowson and Higginson (5) formulas respectively.

$$
\begin{gathered}
\eta=\eta_{o} e^{\left(\log \eta_{o}+9.67\right)\left(\left(1+5.1 \times 10^{-9} p\right)^{Z}-1\right)} \\
\rho=\rho(p)=\rho_{0}\left(\frac{1+\gamma p}{1+\lambda p}\right)
\end{gathered}
$$

To obtain solutions to the elastohydrodynamic problem Eqs. (1) and (2) are solved simultaneously, with Eqs. (3), (4), and (5) used to determine the factors in Eq. (1). Equation (1) is discretised using second order finite elements and Eq. (2) by a central finite difference scheme, and the two equations are expressed in an overall matrix problem whose unknowns are the values of $h$ and $p$ at each node of the computing mesh. The rapid decay of the influence coefficients $f_{i}$ allows the problem to be expressed in a narrow banded form as discussed in [7]. In this way the principal active variables are solved for simultaneously.

In some circumstances contact occurs between the surfaces. If, in a particular timestep a converged result is obtained with a negative calculated film thickness, then the most negative film thickness is set to zero. The hydrodynamic equation corresponding to this nodal point is deleted from the problem matrix, but the elastic deflection equation is retained. (In this way the film thickness value of zero is a boundary condition for the elastic deflection equation which ensures that the pressure distribution obtained remains entirely consistent with the film shape. The pressure developed in the coupled solution at the contacting node is then an automatic boundary condition for the hydrodynamic equation on both sides of the contacting node.) The timestep is then recalculated. This procedure is repeated for the timestep, adding no more than one contact point per timestep re-calculation, until a converged result with no negative calculated film thicknesses is obtained. Contact, as calculated in this way, is found to be a relatively infrequent event in the results obtained as discussed in section 4.3.

A further instance where a full film solution is not obtained at each node point is when what may be termed "in-contact cavitation" occurs. Cavitation at the exit of a contact, where the calculated pressure falls to sub-ambient values, is a natural part of any EHL solution. Situations occur in rough EHL contacts where composite valley features are increasing in volume with time. If flow into such a feature from the micro contacts at either side is insufficient, then the pressure in the composite valley feature will fall. If this effect is sufficiently strong then the oil "trapped" in the composite valley can become entirely decompressed, returning progressively to ambient pressure. If the calculated pressure becomes negative then the lubricant is regarded as cavitated.

Both localized contact and in-contact cavitation are effects determined from the numerical analysis. The sensitivity of these calculated events to the details of the numerical scheme is the focus of a current investigation by the authors that will be reported in due course. There seems to be little doubt that micro contact does indeed take place between rough surfaces in EHL, as electrical contact resistance measurements provide strong supportive evidence of this phenomenon. The occurrence of calculated in-contact cavitation is a new feature of this kind of analysis. Such cavitation may be potentially damaging to the surfaces as it takes place in isolation from the ambient atmosphere.

The high shear rate brought about by sliding in the lubricant film results in energy dissipation and a thermal model has been included in the analysis as reported in [10]. Comparisons of models with and without the thermal analysis have shown that there is little difference in the pressure and film thickness response provided that an allowance is made for inlet zone shear heating. For the current work the simpler isothermal treatment is adopted. The tooth bulk temperatures were not measured in the experiments and the oil feed temperature (an FZG test parameter) is used as a representative temperature value for the purposes of comparison.

The non-Newtonian oil parameter $\tau_{0}$ can be expected to be a function of pressure (and temperature if a thermal model is included). Values of $\tau_{0}$ based on average values over the Hertzian region in traction experiments have been presented by Evans and Johnson [2] and these have shown an increase with increasing pressure for HVI 650, "a mineral oil typical of gear lubricants." Bair [11] however has argued that the linear behavior observed in plotting the EHL traction force against the logarithm of the shear rate is insufficient to establish that Eyring behavior is the appropriate fluid model to use. Bair and Winer have also argued [12] that the Roelands pressure-viscosity form of Eq. (4) may underpredict viscosity at high pressures. The limiting shear stress type behavior advocated by Bair and Winer [3] is very similar to the Eyring model until the limiting state is approached closely, provided that the limiting shear stress parameter is taken to be three times the Eyring stress. The current paper does not seek to contribute directly to this debate, but recognizing that measurements of limiting shear stress in the literature establish it to be proportional to pressure $(\approx 0.05 p)$, the Eyring stress is therefore taken to be given by

$$
\tau_{0}=\kappa p+\frac{\bar{\tau}_{0}^{2}}{\bar{\tau}_{0}+\kappa p}
$$

which gives a dependence resulting in a smooth transition between the constant value of $\bar{\tau}_{0}$ at low pressure to a pressuredependent value $\kappa p$ at high pressure.

\section{Results}

Four different gear tooth surface profile traces taken from standard steel FZG test gears have been used in this work and these are illustrated comparatively in Fig. 1. Roughness parameters evaluated over the parts of the profiles actively modified by surface interaction and used in these analyses are given in Table 1. The profiles are measured by mounting the test gear on a specially manufactured re-location jig for profilometer measurement, which ensures that the same area of the tooth is assessed at each load stage. The $R_{a}$ value for the section of the profile used for the EHL analysis was stable at around $0.33 \mu \mathrm{m}$ for FZG load stages 6,7 , and 8 , and increased by almost 10 percent at the end of load stage 9. The $1.5 \mathrm{~mm}$ portion of the traces shown in Fig. 1 indicates that relocation is achieved effectively as clearly recognisable surface features can be seen in each of the profiles. Inspection confirms the progressive nature of surface modification between the load stages with asperity tips tending to become more rounded. The increase in $R_{a}$ at load stage 9 is probably caused by the increase in 


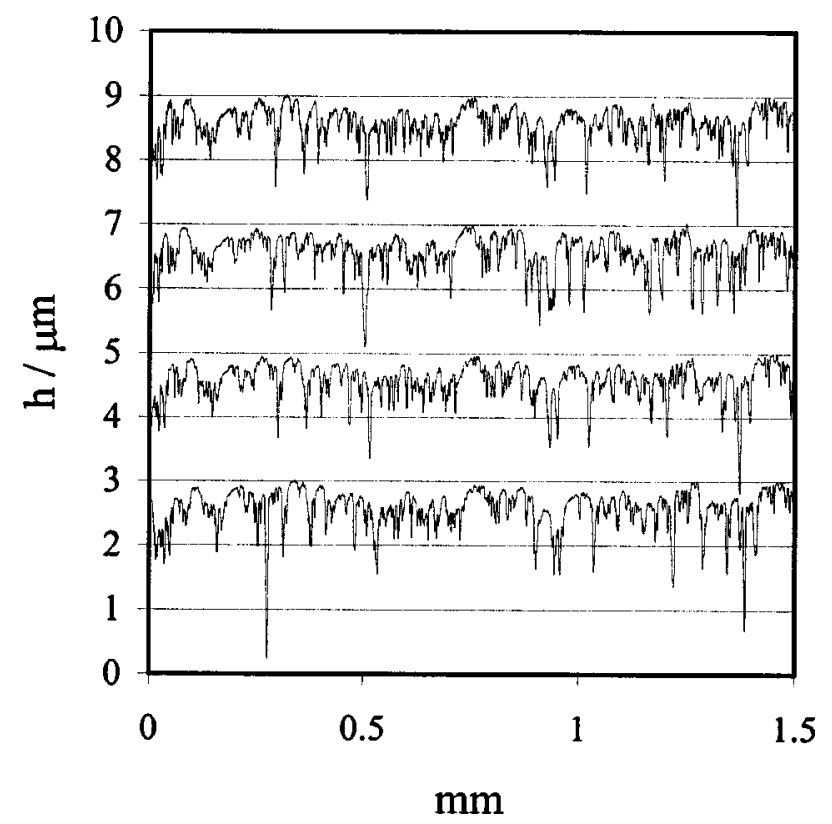

Fig. 1 Profilometer traces for load stages 6 (upper profile), 7, 8 , and 9 (lower profile) used in the analyses each offset by 2 $\mu \mathrm{m}$ from neighboring profiles for comparison. Profiles are drawn with metal below the curve.

micropitting observed after that load stage with some micropitted features being included in the assessed profile, although it is recognized that $R_{a}$ is not a particularly reliable parameter for differentiation of subtle surface changes. The lubricant modelled is a medium viscosity mineral oil containing a mixed complex of phosphorus and sulphur extreme pressure additives used in ship propulsion gearboxes which have high gear tooth loading. For the current work the viscosity at atmospheric pressure is taken to be $\eta_{0}=0.028 \mathrm{Pas}$, and the pressure viscosity coefficient used to determine parameter $Z$ in Eq. (4) is $\alpha=17.5 \mathrm{GPa}^{-1}$. The profiles were acquired from test gears run using this oil at a pinion speed of $2250 \mathrm{rpm}$ and a test temperature of $60^{\circ} \mathrm{C}$, and the four conditions during the meshing cycle identified for analysis are given in Table 2.

In the current paper three different expressions for the Eyring stress $\tau_{0}$ are considered as specified by the parameter values for $\bar{\tau}_{0}$ and $\kappa$ which are given in Table 3 . Model A is the standard

Table 1 Surface profile parameters and nominal contact pressure for the profiles used

\begin{tabular}{|c|c|c|c|}
\hline Load Stage & $\begin{array}{c}\text { Contact } \\
\text { pressure } \\
/ \mathrm{GPa}\end{array}$ & $\begin{array}{c}\mathrm{R}_{\mathrm{a}} \\
/ \mu \mathrm{m}\end{array}$ & $\begin{array}{c}\text { Load } \\
/ \mathrm{N}\end{array}$ \\
\hline 6 & 0.945 & 0.333 & 240 \\
\hline 7 & 1.094 & 0.328 & 321 \\
\hline 8 & 1.245 & 0.328 & 416 \\
\hline 9 & 1.395 & 0.358 & 523 \\
\hline
\end{tabular}

Table 2 Mesh conditions analyzed

\begin{tabular}{|c|c|c|c|c|c|c|}
\hline Mesh Position & Load share & $\begin{array}{c}\text { Load } \\
/ \mathrm{kNm}^{-1}\end{array}$ & $\begin{array}{c}R \\
/ \mathrm{mm}\end{array}$ & $\begin{array}{c}\mathrm{p}_{\mathrm{Hz}} \\
/ \mathrm{GPa}\end{array}$ & $\begin{array}{c}\bar{U} \\
/(\mathrm{m} / \mathrm{s})\end{array}$ & $\xi$ \\
\hline 1 & $60 \%$ & 158 & 6.8 & 0.838 & 3.11 & -0.6 \\
\hline 2 & $100 \%$ & 264 & 8.0 & 1.094 & 3.23 & -0.2 \\
\hline 3 & $100 \%$ & 264 & 8.7 & 0.992 & 3.39 & 0.3 \\
\hline 4 & $60 \%$ & 158 & 8.3 & 0.758 & 3.58 & 0.8 \\
\hline
\end{tabular}

Table 3 Non-Newtonian parameter specification

\begin{tabular}{|c|c|c|}
\hline Model & $\begin{array}{c}\bar{\tau}_{0} \\
/ \mathrm{MPa}\end{array}$ & $\kappa$ \\
\hline $\mathrm{A}$ & 10 & 0.030 \\
\hline $\mathrm{B}$ & 10 & 0.015 \\
\hline $\mathrm{C}$ & 10 & 0 \\
\hline
\end{tabular}

model considered in analysing the rough surfaces, and the results for all three models are compared for the conditions of load stage 9.

Comparison of the Load Stages. Each of the four profiles was taken in turn and simulated in "rough on rough" contact conditions at the standard operating conditions which were taken to be $R=9.7 \mathrm{~mm}, U_{1}=3.90 \mathrm{~m} / \mathrm{s}$ and $U_{2}=2.88 \mathrm{~m} / \mathrm{s}$, i.e., $\xi=0.3$. The EHL problem was analyzed with a grid dimension $\Delta x$ $=a / 400$, and a time step $\Delta t$ chosen such that the faster moving surface passed through a distance $0.25 \Delta x$ in each timestep. The sensitivity to mesh size of the results obtained using this model has been discussed and illustrated in [7] where a similar real roughness profile was analyzed using mesh spacings of $\Delta x$ $=a / 384, \Delta x=a / 192, \Delta x=a / 96$. The results obtained showed that there were no significant differences in the predicted film thickness profiles as the mesh was varied. The mesh adopted for the current work is slightly finer $(a / 400)$.

The surface height data at the end of each test load stage were obtained using a Talysurf profilometer instrument. Intermediate height values, as required in the EHL analysis, were obtained for each timestep by cubic spline interpolation. Each analysis started from the corresponding smooth surface steady state solution, and the rough surfaces were then allowed to migrate from the entry boundary, located at $x=-3 a$, to the exit at the speeds of the respective surfaces. Some 7000 timesteps were considered from the time at which the roughness of the slower moving surface first reached the exit boundary at $x=1.5 a$. Parameters were evaluated from the pressure and film thickness distributions for the rough on rough contact during these latter timesteps. These parameters were introduced in [10] and provide measures of extreme pressure behavior, extreme asperity proximity, and pressure cycling within the contact.

Pressure and film thickness output for specific timesteps are presented in Fig. 2 and 3 for load stages 6 and 9, respectively. The two rough surfaces are shown in their current contact conditions beneath the pressure distribution so that the film thickness is given by the distance between the two rough surfaces. Also shown beneath the pressure and film thickness curves is a contour plot of the subsurface maximum shear stress corresponding to the current pressure distribution. Both figures show that the pressure distribution is made up of a series of elevated pressure areas separated by areas with lower pressure values that remain at a substantial level. The elevated pressure regions are wider than the roughness features and typically correspond to relatively rounded "run-in" land features. The maximum corresponding Hertzian pressure for load stage 6 is $0.95 \mathrm{GPa}$, and that for load stage 9 is $1.40 \mathrm{GPa}$, so it is clear that pressures well in excess of these values are generated on individual micro asperity contacts. Pressure values in the composite valley areas are much lower, but it is also clear that in both these examples the pressurised valley features contribute significantly to the load carrying of the overall EHL film. The elevated values and rapid spatial variation of pressure leads to concentrations of shear stress levels close to the surface. In Fig. 2, the highest value of maximum shear stress is about $0.42 \mathrm{GPa}$, which may be compared with a subsurface value of $0.28 \mathrm{GPa}$ for the corresponding smooth surface case. For load stage 9, as shown in Fig. 3, maximum shear stress values as high as $0.7 \mathrm{GPa}$ occur compared with the smooth surface value of $0.42 \mathrm{GPa}$. Video sequences made up of such figures enable the variation of pressure 

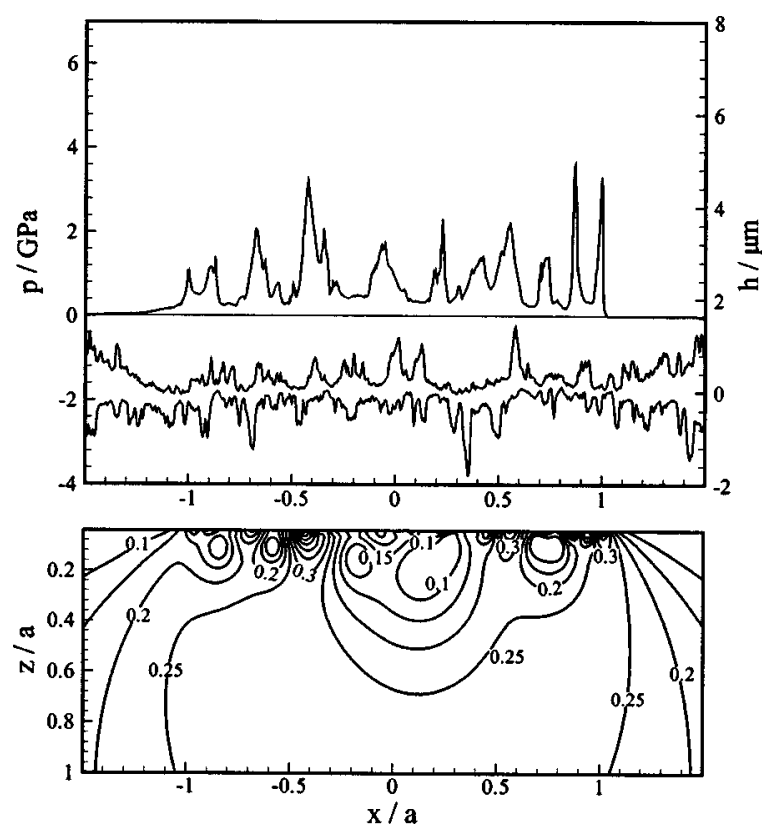

Fig. 2 Pressure and film thickness variation at a timestep for load stage 6 with Model A. Also shown below are contours of $\pi \mathrm{GPa}$ for the sub-surface maximum shear stress.

and film thickness to be assessed in a qualitative way and are invaluable for developing an understanding of the mechanisms at work as individual asperity features enter the Hertzian contact area and subsequently pass through it, as discussed in [7]. Videos for the results presented in this paper are available from the authors on $\mathrm{CD}$ by request. Contact between individual asperities and internal cavitation have been observed in such numerical solutions, as discussed in [10] where a thinner oil $\left(\eta_{0}\right.$ $=0.0048 \mathrm{Pas}, \alpha=11.1 \mathrm{GPa}^{-1}$ ) was used for the analysis. Since the surfaces are different and loaded to different extents it is not possible to compare like with like at the individual timestep level.
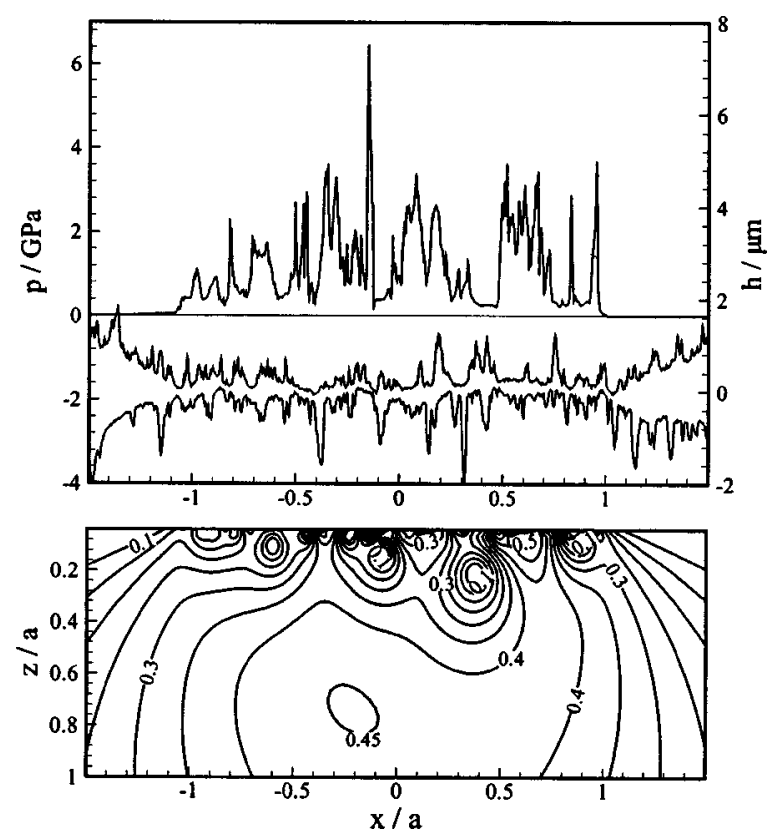

Fig. 3 Pressure and film thickness variation at a timestep for load stage 9 with Model A. Also shown below are contours of $\pi \mathrm{GPa}$ for the sub-surface maximum shear stress.

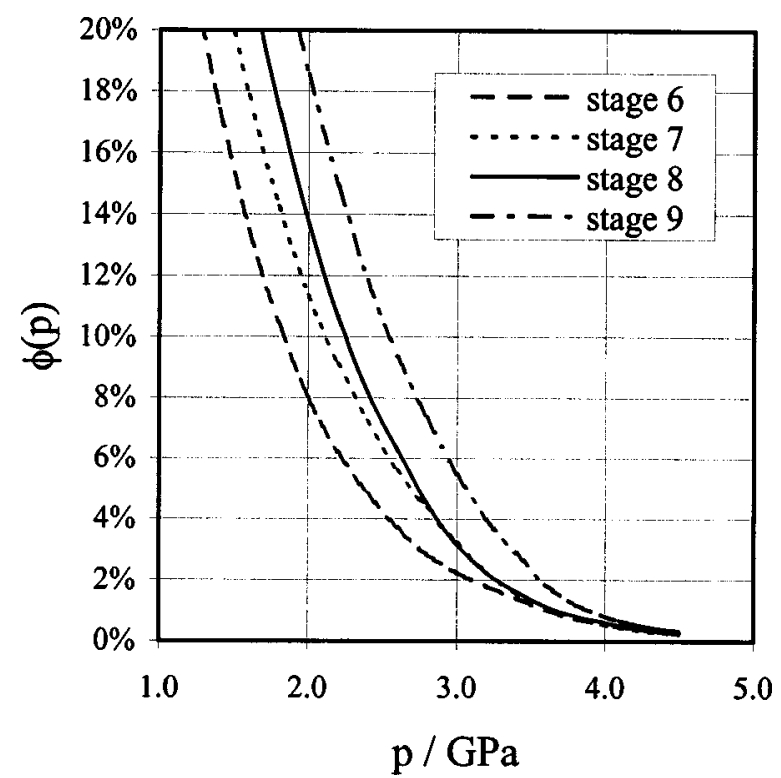

Fig. 4 High pressure behavior curves of the four load cases each averaged over 7000 timesteps

However, average behavior parameters can be compared, and for the current comparisons averages taken over a period of 7000 timesteps have been calculated. During this time the faster moving surface moves by a distance of more than 4 times the Hertz contact dimension.

Figure 4 shows the extreme high pressure behavior for the Hertzian area of the four load cases considered. Parameter $\Phi(p)$ is the fraction of the Hertzian contact area that has a pressure that exceeds $p$. Load stage 6 has the least amount of its contact area subject to extremes of pressure, and indeed load stage 9 has the greatest amount. However all cases tend to the same level of extreme pressure with about 0.25 percent of the area subject to pressures of $4.5 \mathrm{GPa}$. The maximum pressures are thus seen to be significant in relation to the hardness of the gear teeth (700 Vickers hardness number) suggesting that surface modification is achieved by plastic deformation under the action of the EHL asperity pressures. The fraction of the contact area subject to pressures between 1 and $2 \mathrm{GPa}$ is seen to rank the profiles according to the applied load, whereas true extremes of pressure may well be governed by surface hardness considerations. Figure 5 gives the cumulative film thickness distribution for small film thickness values for the four profiles. Parameter $\Phi(h)$ is the fraction of the Hertzian contact area that has a film thickness that is less than $h$. The lowest film values observed are of the order $0.075 \mu \mathrm{m}$, and the earlier load stages have a greater tendency towards the lower film values, for example 2 percent of the contact area for load stage 6 is below $0.15 \mu \mathrm{m}$, a figure that reduces to 1 percent for load stage 9 . Figures 4 and 5 thus suggest that the surface modification (running in) induced at each load stage causes the rough surface to adopt a shape that is more able to generate lubricant films on the micro asperities. Figure 6 quantifies the pressure cycling behavior of the different load stages. The curves indicate the cycle counts obtained between two specified pressure limits. The count is the number of times within the Hertzian contact area that pressure exceeds the specified upper pressure limit and subsequently falls below the lower pressure limit in an individual timestep. A lower pressure limit of $0.5 \mathrm{GPa}$ was used for all the results in this paper, and variation of the upper pressure limit used for the count gives rise to the curves shown in Fig. 6, for example. The counts can be seen to vary systematically, with load stage 9 having the greater number of pressure cycles. However, if the counts are normalized to the number of cycles per unit length the 


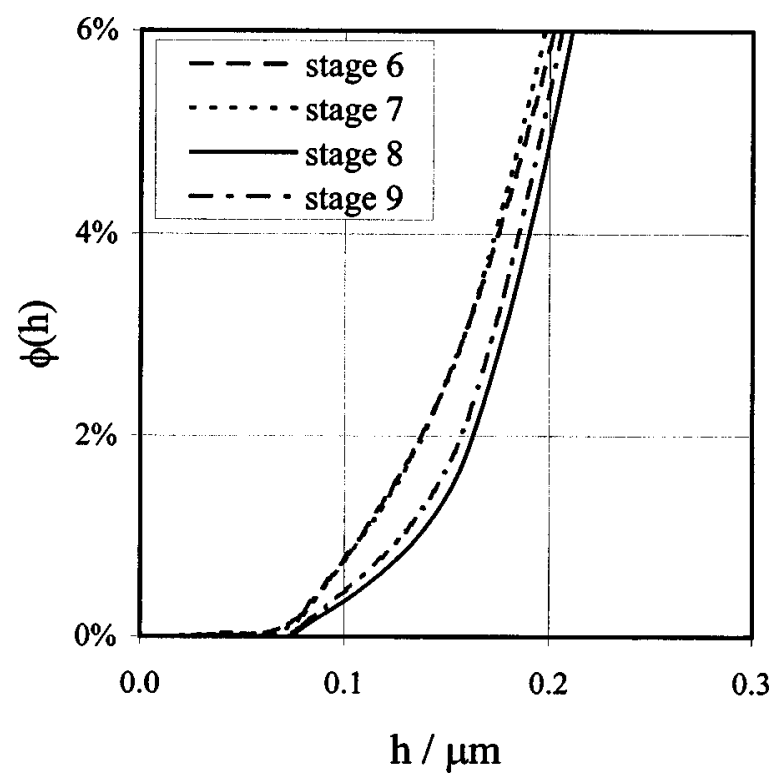

Fig. 5 Low film thickness behavior curves for the four load cases each averaged over 7000 timesteps

curves become closer to each other and cross, so that the order is reversed for counts obtained using the smaller values of the upper pressure limit.

Variation Over the Meshing Cycle. The second set of comparisons made are between models using roughness profiles from load case 7 over the range of conditions that occur within the meshing cycle of the gear pair. The conditions analyzed are specified in Table 2. Mesh position 1 is in the load sharing stage prior to single tooth loading; mesh positions 2 and 3 have single tooth loading and are located on either side of the pitch point; and mesh position 4 is in the shared loading region as the tooth pair moves out of mesh. For mesh positions 1 and 4 the high sliding speed gives high entrainment factors for the individual asperity contacts within the Hertzian region, an influence associated with higher asperity film thicknesses and pressures when varied in isolation

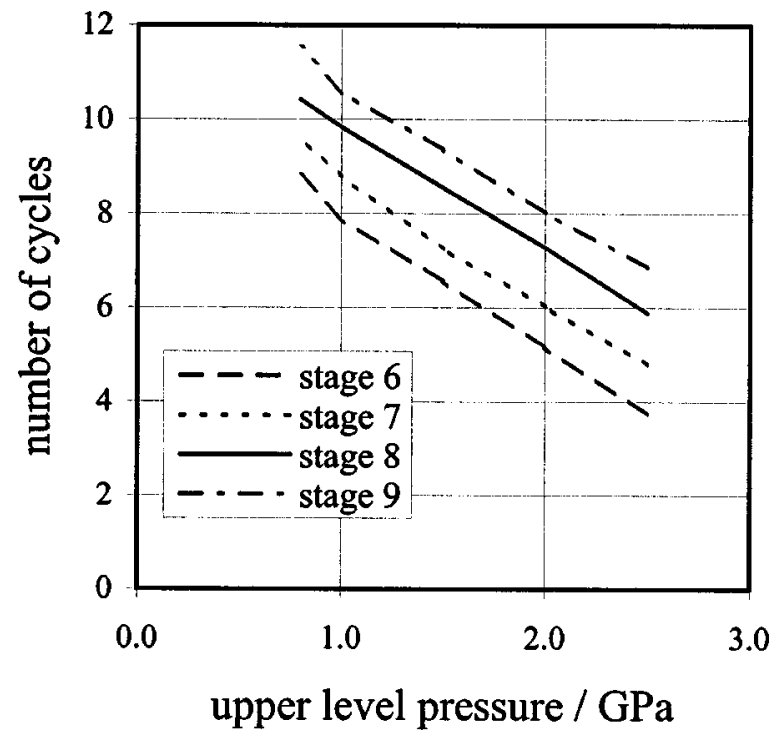

Fig. 6 Pressure cycle counts obtained for the four load cases for a lower pressure cycle limit of $0.5 \mathrm{GPa}$, each averaged over 7000 timesteps

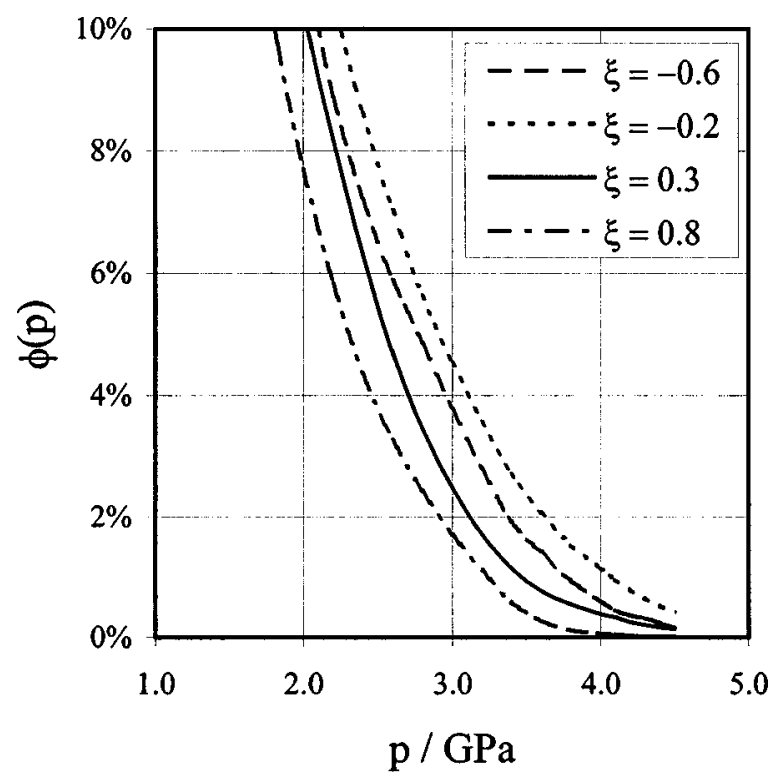

Fig. 7 High pressure behavior curves for the cases considered over the meshing cycle

[7]. In contrast the full load mesh positions have lower sliding but, as seen for the load stage comparisons, higher load is also associated with higher asperity film thicknesses and pressures. To some extent, therefore, the influence of load reduction at the beginning and end of the contact path is compensated for by the increased sliding. Figure 7 shows the differences in extreme pressure behavior between the conditions as the contact moves through the gear mesh and Fig. 8 the corresponding thin film behavior. There is no clear trend as both sliding and load vary over the meshing cycle, but for each of the loads, the case with higher sliding has smaller extreme pressures and thicker extreme film thicknesses. The pressure cycle count shown in Fig. 9 does rank the cases according to sliding speed with the highest pressure cycling observed at the full load/lowest sliding case. This ranking is preserved if the count is normalized to distance, but the differences between full and $60 \%$ load then become less pronounced.

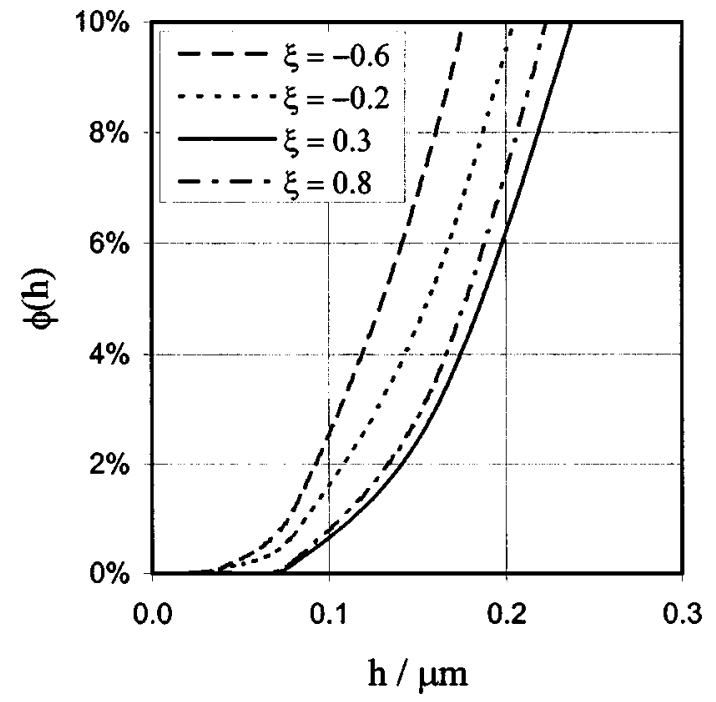

Fig. 8 Low film thickness behavior curves for the cases considered over the meshing cycle 


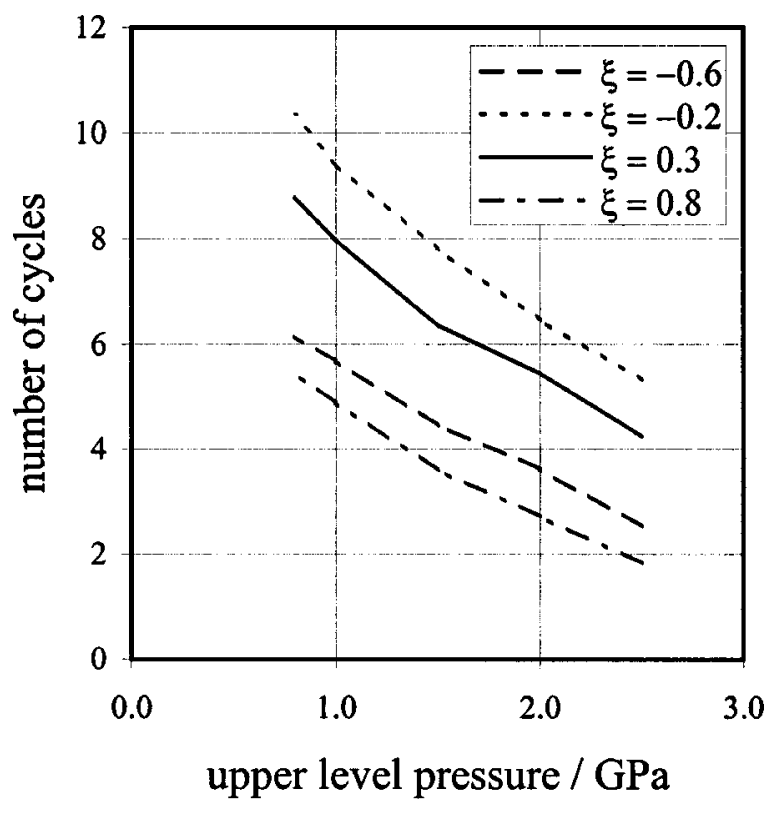

Fig. 9 Pressure cycle counts obtained for the cases considered over the meshing cycle for a lower pressure cycle limit of $0.5 \mathrm{GPa}$

Comparison of Non-Newtonian Models. Load case 9 was examined using three different expressions for the non-Newtonian parameter $\tau_{0}$ as specified in Table 3. All models have the same value of $\tau_{0}$ at low pressures, but models A and B have a $\tau_{0}$ value that increases with pressure, with a greater rate of increase for model A. The comparisons illustrate the sensitivity of the numerical results to the non-Newtonian behavior assumed. Limiting shear stress effects have not been included in the current paper and will form the focus of a further contribution in due course.

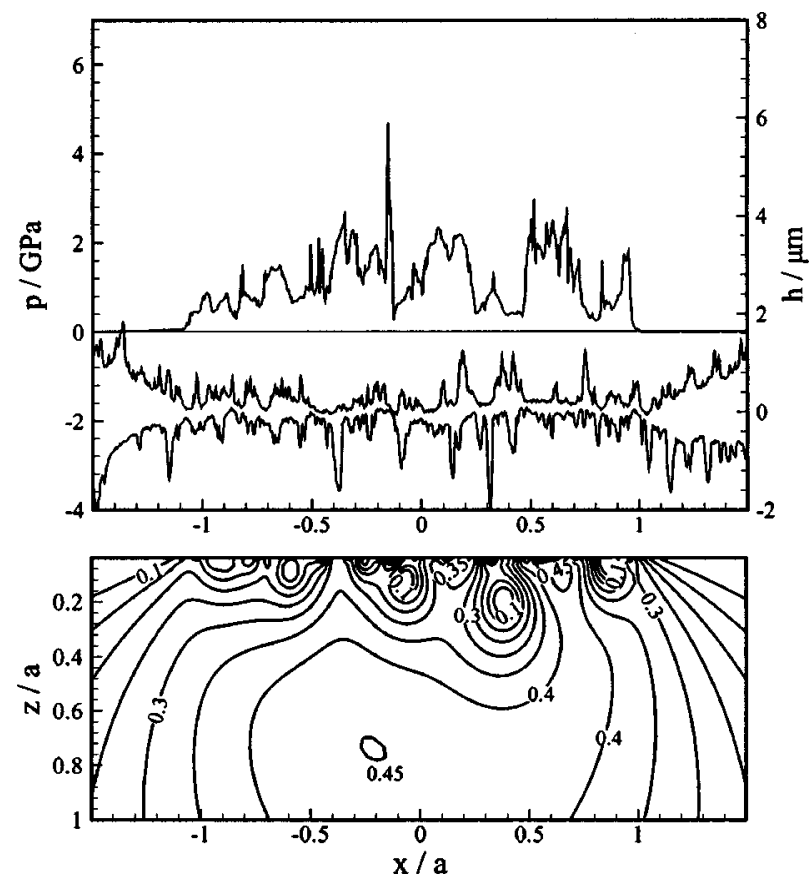

Fig. 10 Pressure and film thickness variation at a timestep for load stage 9 with Model C. Also shown below are contours of $\pi \mathrm{GPa}$ for the sub-surface maximum shear stress.

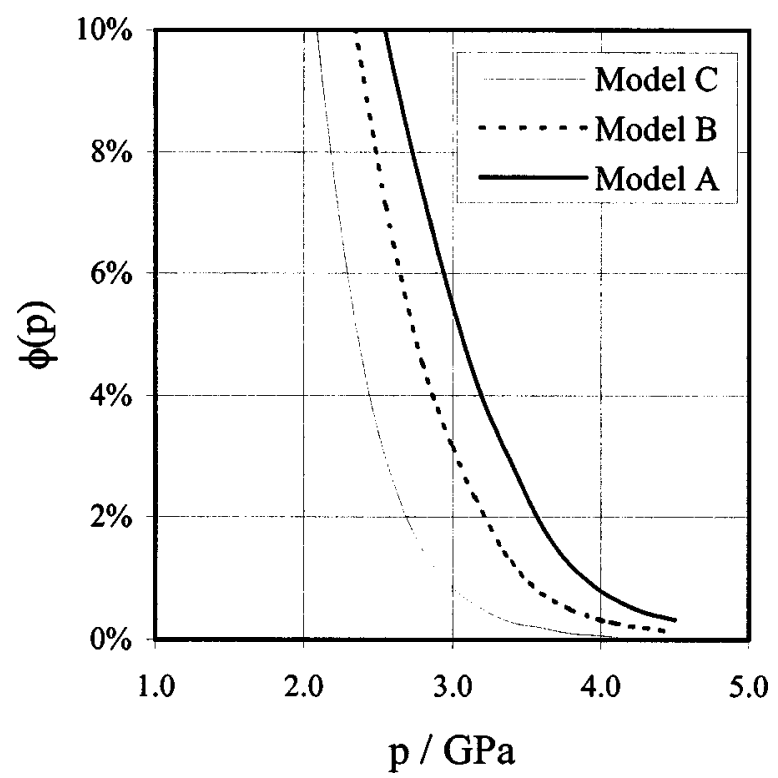

Fig. 11 High pressure behavior curves for the three nonNewtonian models with load case 9

Figure 10 gives the pressure, film thickness, and subsurface shear stress distributions for Model $\mathrm{C}$ and gives a direct comparison with Fig. 3 where Model A is used for the same case. Inspection of these figures suggests that Model A, which has the highest values of $\tau_{0}$ at elevated pressures can be seen to cause higher peak pressures together with thicker films on asperity micro contacts. Model C, where $\tau_{0}$ is constant, is seen to have a greater degree of load sharing between the micro asperity contacts and the remainder of the contact region. The pressures developed at micro contacts are lower, as too is the film thickness seen there. The maximum sub-surface shear stress seen in this timestep with Model C is $0.45 \mathrm{GPa}$, compared with $0.7 \mathrm{GPa}$ for Model A, so that the non-Newtonian behavior is seen to have a big influence on the subsurface shear stress developed near the surface. Indeed, for the timestep shown in Fig. 10, the maximum shear stress is no higher than that occurring with smooth surfaces. Model B, which lies between models $\mathrm{A}$ and $\mathrm{C}$ in pressure sensitivity, is found to produce an intermediate outcome in comparison with the other two models. The high $\tau_{0}$ values produced by Model A inhibit the shear thinning behavior and keep the effective viscosity relatively high in the high pressure, high pressure gradient regime. Model $\mathrm{C}$, in contrast, allows the pressure-driven flow to actively change the oil's position, flowing away from the micro asperity contacts as they occur due to the sliding motion, and pressurising the valleys through the lubricant's compressibility behavior. The behavior shown in this individual timestep comparison is borne out in the average cycle behavior. Figure 11 confirms that Model A leads to consistently higher micro asperity pressure levels, and Fig. 12 shows that this is associated with higher micro asperity film thicknesses. Pressure cycle counts, shown in Fig. 13, also confirm that the EHL response for the lubricant of Model A is considerably more aggressive than that of Model $\mathrm{C}$.

Tables 4, 5, and 6 quantify the occurrences of surface to surface contact and of cavitation within the nominal contact region for the cases considered. The number of timesteps in which these situations occur is expressed as a percentage of the total, and the average number of mesh points involved in the events is also tabulated. In general contact is seen to be a much rarer calculated event than is cavitation. Over the range of models considered contact takes place for 0.25 percent of the total number of timesteps, and this proportion is no more than 1.1 percent of timesteps for the lowest load case model, which shows the greatest tendency to contact amongst those considered. Contact is seen 


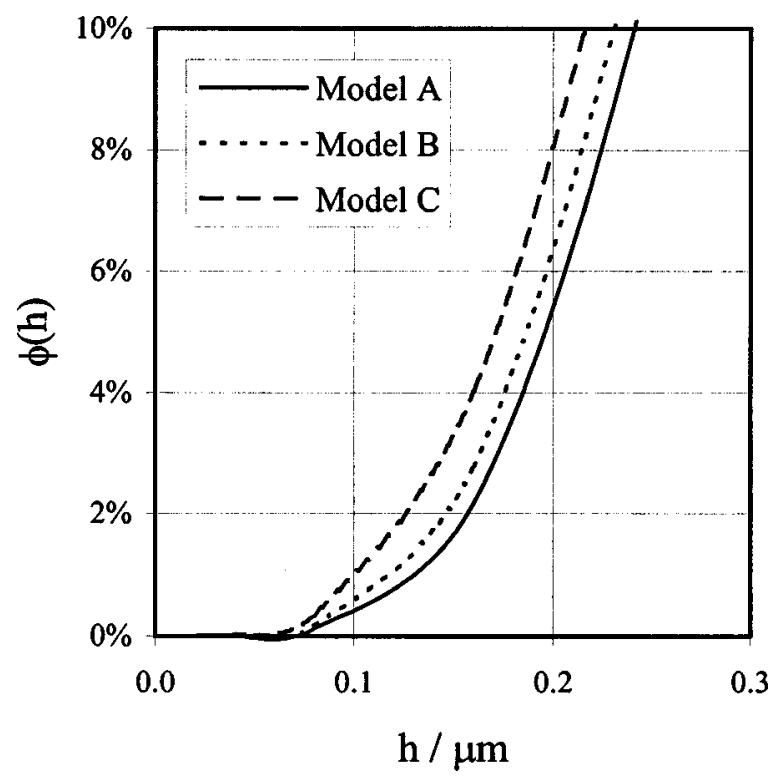

Fig. 12 Low film thickness behavior curves for the three nonNewtonian models with load case 9

to take place predominantly at one mesh point when it occurs. Since the surfaces are being successively modified at each load stage it may well be the case that a high degree of contact is a feature of raw, as-manufactured profiles, and that the running-in process reduces its occurrence to a very low background level. Its

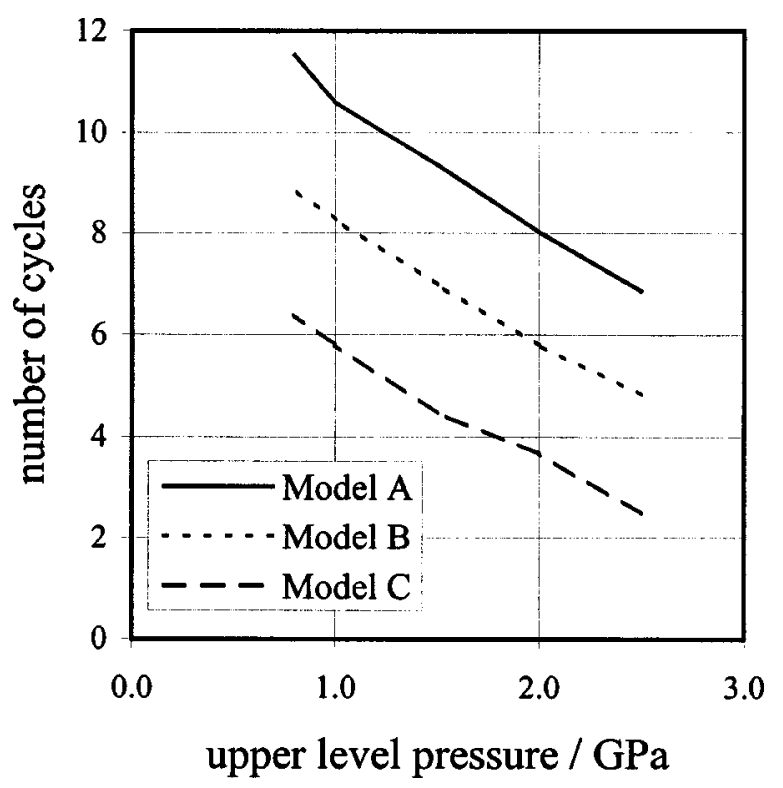

Fig. 13 Pressure cycle counts obtained for a lower pressure cycle limit of $0.5 \mathrm{GPa}$ for the three non-Newtonian models with load case 9

Table 4 Summary of contact and cavitation events over $\mathbf{7 0 0 0}$ timesteps for load case comparisons

\begin{tabular}{|c|c|c|c|c|}
\hline $\begin{array}{c}\text { Load } \\
\text { Case }\end{array}$ & $\begin{array}{c}\text { Timesteps } \\
\text { with } \\
\text { contact }\end{array}$ & $\begin{array}{c}\text { Contacting } \\
\text { points }\end{array}$ & $\begin{array}{c}\text { Timesteps } \\
\text { with } \\
\text { cavitation }\end{array}$ & $\begin{array}{c}\text { Cavitation } \\
\text { points }\end{array}$ \\
\hline 6 & $1.1 \%$ & 1.0 & $7.4 \%$ & 8.8 \\
\hline 7 & $0.23 \%$ & 1.06 & $3.4 \%$ & 3.4 \\
\hline 8 & 0 & 0 & 0 & 0 \\
\hline 9 & $0.21 \%$ & 1.13 & $6.5 \%$ & 4.3 \\
\hline
\end{tabular}

Table 5 Summary of contact and cavitation events over 7000 timesteps for meshing cycle comparisons

\begin{tabular}{|c|c|c|c|c|}
\hline $\begin{array}{c}\text { Mesh } \\
\text { Position }\end{array}$ & $\begin{array}{c}\text { Timesteps } \\
\text { with } \\
\text { contact }\end{array}$ & $\begin{array}{c}\text { Contacting } \\
\text { points }\end{array}$ & $\begin{array}{c}\text { Timesteps } \\
\text { with } \\
\text { cavitation }\end{array}$ & $\begin{array}{c}\text { Cavitation } \\
\text { points }\end{array}$ \\
\hline 1 & $0.1 \%$ & 1.0 & $25.7 \%$ & 46.8 \\
\hline 2 & $0.4 \%$ & 1.0 & $6.5 \%$ & 2.4 \\
\hline 3 & $0.01 \%$ & 1.0 & $6.9 \%$ & 7.9 \\
\hline 4 & $0.1 \%$ & 1.0 & $22.2 \%$ & 32.2 \\
\hline
\end{tabular}

Table 6 Summary of contact and cavitation events over 7000 timesteps for non-Newtonian model comparisons

\begin{tabular}{|c|c|c|c|c|}
\hline$\tau_{0}$ model & $\begin{array}{c}\text { Timesteps } \\
\text { with } \\
\text { contact }\end{array}$ & $\begin{array}{c}\text { Contacting } \\
\text { points }\end{array}$ & $\begin{array}{c}\text { Timesteps } \\
\text { with } \\
\text { cavitation }\end{array}$ & $\begin{array}{c}\text { Cavitation } \\
\text { points }\end{array}$ \\
\hline Model C & $0.36 \%$ & 1.0 & $7.2 \%$ & 2.61 \\
\hline Model B & $0.07 \%$ & 1.0 & $6.6 \%$ & 2.69 \\
\hline Model A & $0.21 \%$ & 1.13 & $6.5 \%$ & 4.30 \\
\hline
\end{tabular}

prevalence is associated with the shape of asperity tip features which will be the subject of modification by plastic deformation.

Cavitation, on the other hand, is associated with asperity valley features and is caused where a composite valley is formed whose volume is increasing with time. The composite valley is bounded by asperity tip micro contacts, and if the flow into the valley at these micro contacts is insufficient, its pressure will drop and may ultimately fall below ambient levels, causing cavitation. Mesh positions 1 and 4 in Table 5 show that cavitation is more frequent when sliding is high, as the high sliding conditions of mesh positions 1 and 4 (Table 5) have much higher proportions of cavitation than do mesh positions 2 and 3. These cavitation events also take place over a much larger number of mesh points in high sliding circumstances. It should be borne in mind, however, that due to the reduced load in the load-sharing region of the meshing cycle the spatial resolution for these high sliding cases is finer, with $\delta x$ reduced by a factor of 0.71 in comparison to the full load mesh positions.

\section{Conclusions}

- Comparisons made in the paper show that extreme conditions of micro asperity pressures and film thicknesses vary with load. Use of experimental data from different load stages of an FZG gear test suggest that the surface modification (running-in) taking place is such as to limit the pressure extremes to a similar level in spite of the varying load.

- Comparison of conditions representative of different parts of the meshing cycle indicate that the increase in sliding at the extremes of the cycle may be an aid to micro film formation and thereby reduce surface vulnerability that would otherwise be caused by the lower viscosity due to the reduced load. Increased sliding leads to a greater incidence of calculated cavitation, however, and this may be of considerable significance per se in relation to micropitting.

- Significant differences are seen in extreme asperity film and pressure levels as different non-Newtonian parameter expressions are compared. This observation emphasises the importance of being able to establish experimentally which of a range of such models is most appropriate.

- Comparisons have been made using surface profiles from the different load stages. In reality different parts of each tooth profile encounter specific parts of the mating profile(s) with specific load and kinematic conditions. The different tendencies seen in these preliminary investigations may well be more clear-cut if the true meshing cycle is modelled in terms of actual contacting profiles, load sharing, sliding, entrainment and radius of relative curvature as these change along the path of contact. 


\section{Acknowledgments}

The authors gratefully acknowledge the support of the Engineering and Physical Science Research Council (Grant GR/ L90996) that allowed this research to be undertaken, along with financial support from the British Gear Association. The authors freely acknowledge the many helpful discussions held with collaborating parties to the BGA Understanding Micropitting project.

\section{Nomenclature}

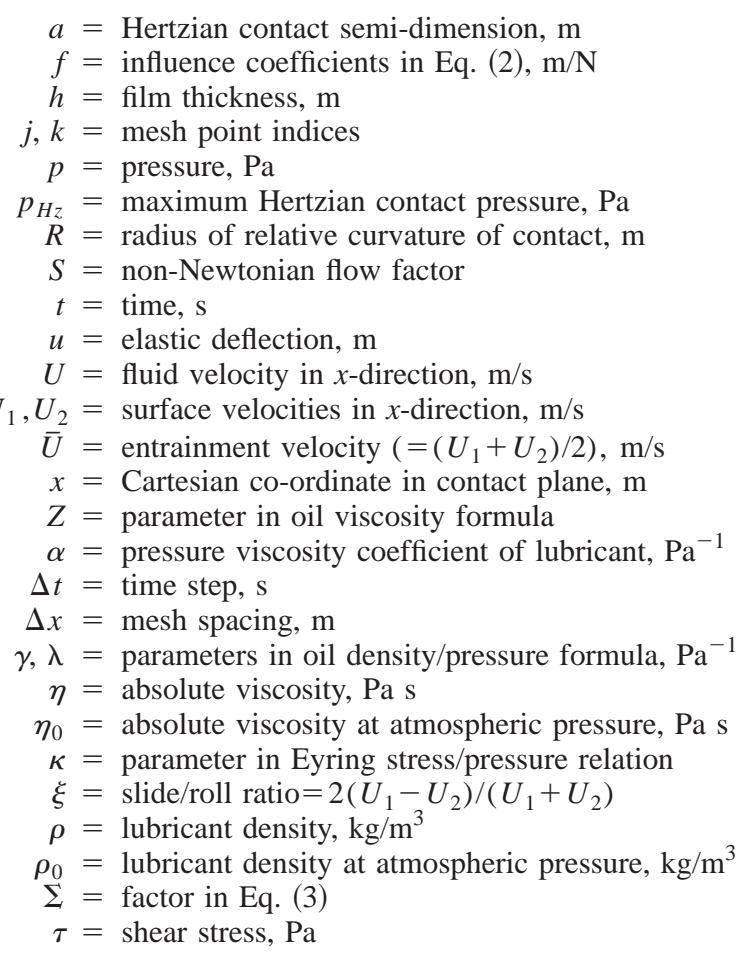

$\tau_{0}=$ Eyring shear stress, $\mathrm{Pa}$

$\widetilde{\tau}_{0}=$ Eyring shear stress at low pressure, $\mathrm{Pa}$

$\phi=$ rough surface profile height function in Eq. (2), $\mathrm{m}$

$\Phi(h)=$ fraction of dry contact area whose film thickness is less than $h$.

$\Phi(p)=$ fraction of dry contact area whose pressure exceeds $p$

\section{References}

[1] Johnson, K. L., and Tevaarwerk, J. L., 1977, "The Shear Behavior of Elastohydrodynamic Oil Films," Proc. R. Soc. London, Ser. A, A356, pp. 215-236.

[2] Evans, C. R., and Johnson, K. L., 1986, "The Rheological Properties of Elastohydrodynamic Lubricants," Proc. Inst. Mech. Eng., Part C: Mech. Eng. Sci., 200, pp. 303-312.

[3] Bair, S., and Winer, W. O., 1979, "A Rheological Model for Elastohydrodynamic Contacts Based on Primary Laboratory Data," ASME J. Lubr. Technol., 101, pp. $258-265$.

[4] Zhu, D., and Hu, Y. Z., 1999, "The Study of Transition From Full Film Elastohydrodynamic to Mixed and Boundary Lubrication," Proc. STLE/ASME, H. S. Cheng ed., Tribology Surveillance, pp. 150-156.

[5] Hu, Y. Z., and Zhu, D., 2000, "A Full Numerical Solution to the Mixed Lubrication in Point Contacts,” ASME J. Tribol., 122, pp. 1-9.

[6] Zhu, D., and Hu, Y. Z., 2000, "Effects of Rough Surface Topography and Orientation on the EHD and Mixed Lubrication Characteristics," Proceedings International Tribology Conference, Nagasaki, pp. 625-630.

[7] Elcoate, C. D., Hughes, T. G., Evans, H. P., and Snidle, R. W., 2001, "Transient Elastohydrodynamic Analysis Using a Novel Coupled Differential Deflection Method," Proc. Inst. Mech. Eng., Part J: J. Eng. Tribol., 215, pp. 319-337.

[8] Evans, H. P., and Hughes, T. G., 2000, "Evaluation of Deflection in SemiInfinite Bodies by a Differential Method," Proc. Inst. Mech. Eng., Part C: Mech. Eng. Sci., 214, pp. 563-584.

[9] Conry, T. F., Wang, S., and Cusano, C., 1987, “A Reynolds-Eyring Equation for Elastohydrodynamic Lubrication in Line Contacts,” ASME J. Tribol., 109, pp. $648-654$

[10] Tao, J., Hughes, T. G., Evans, H. P., and Snidle, R. W., 2002, "Elastohydrodynamic Response of Transverse Ground Gear Teeth," Proc. 28th Leeds-Lyon Symp. on Tribology, Elsevier, Amsterdam, pp. 447-458.

[11] Bair, S., 2000, "Pressure-Viscosity Behavior of Lubricants to $1.4 \mathrm{GPa}$ and Its Relation to EHD Traction," Tribol. Trans., 43, pp. 91-99.

[12] Bair, S., and Winer, W. O., 2000, "The Pressure-Viscosity Coefficient at Hertz Pressure and Its Relation to Concentrated Contact Traction," Proc. 26th LeedsLyon Symp. on Tribology, Elsevier, pp. 433-444. 